\title{
A two kinase-gene signature model using CDK2 and PAK4 expression predicts poor outcome in non-small cell lung cancers
}

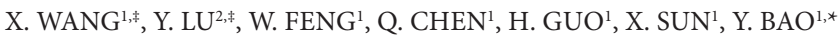 \\ ${ }^{1}$ Department of Gastrointestinal Surgery, First Affiliated Hospital, Huzhou Teachers College, the First People's Hospital of Huzhou, Huzhou \\ 313000, China; ${ }^{2}$ Department of Medicine, Huzhou Teachers College, Huzhou 313000, China \\ ${ }^{*}$ Correspondence: baoying2222@126.com \\ ${ }^{*}$ Contributed equally to this work.
}

Received August 17, 2015 / Accepted October 26, 2015

\begin{abstract}
Risk classification on the basis of specific genomic features can lead to more precise tailoring of treatment for cancer patients. Kinases are potential therapeutic targets and survival factors, but the predictive prognostic potentials of multi-kinase genes have seldom been investigated. In this study, with publicly available microarray data of non-small cell lung cancers (NSCLC), we identified two kinase genes cyclin-dependent kinase 2 (CDK2) and p21 protein (Cdc42/Rac)-activated kinase 4 (PAK4) significantly associated with poor outcome. Then we present a combined gene signature model using CDK2 and PAK4 that can stratify disease poor outcome independently of standard clinical prognostic factors. Next, the predictive robustness of this 2-gene classifier was in silico confirmed in an independent microarray dataset, and experimentally validated in a lung cancer cohort by immunohistochemistry. Therefore, in this study, we demonstrated that the CDK2-PAK4 kinase signature may be a useful prognostic indicator and potential target for NSCLC. We also propose that poor outcome subgroup stratified by this classifier may benefit from the recently developed CDK2 and PAK4 inhibitors.
\end{abstract}

Key words: kinase, non-small lung cancer, gene signature, CDK2, PAK4

Lung cancer is the leading cause of cancer death globally. Non-small cell cancer (NSCLC), which comprises more than $80 \%$ of lung cancers, generally has a relatively poor clinical outcome. Unfortunately, the current clinicopathological staging system is not adequate to risk-stratify NSCLC patients, which seem to be due to the heterogeneity of the gene expression patterns among cancers. For cancer harboring multiple genetic abnormalities, multi-gene signature would usually provide superior information to single marker in prognosis prediction.

Recently, based on the gene expression profile, a series of gene signatures have been developed for the risk classification in NSCLC [1-3]. However, the results of these gene expression studies in NSCLC have usually been inconsistent [4]. To date, no established gene signature has been widely used in clinical practice. Additionally, most of the current gene signatures lack biological meaning, which makes it difficult for investigators to interpret therapeutic significance.

Kinases are well established as a validated and promising drug target class for cancer treatment, especially, inhibitors against some kinases, such as epidermal growth factor receptor (EGFR), anaplastic lymphoma kinase (ALK), have succeed in the clinical treatment of select NSCLC patients [5]. Currently, more than 500 human kinase genes have been identified or predicted [6]. Until now, the prognostic significance of several kinase genes has been widely investigated in lung cancer [7-8]. However, most of these studies focused on single kinase genes, and in single-centered analysis. The prognostic performance of the kinase genes for NSCLC patients has not been compared. In particular, the use of combined kinase gene signature in predicting NSCLC survival has not yet been reported in previous studies.

We hypothesized that a kinase gene expression signature may serve as a more powerful prognostic factor than single kianse genes for outcome in NSCLC. In this study, by comparing the prognostic potentials of kinase genes across several microarray dataset of NSCLC, we developed and experimentally validated a new two-kinase gene (CDK2-PAK4) signature that can stratify disease outcome independently of standard clinical prognostic factors. Our findings also highlighted the 
importance of this kinase gene signature for mechanism of tumor progression and therapeutic intervention in NSCLC cancer.

\section{Materials and methods}

Gene expression microarray datasets for survival analysis. To systemically compare the predictive powers of kinase genes for the outcome, we used 3 publicly available lung cancer microarray datsets (GSE11969, GSE26939, and GSE30219) from the GEO database. GSE1 1969 dataset, derived from the Agilent Homo sapiens 21.6K custom array platform, comprises the expression data of 18,175 unique genes of 149 NSCLC samples, 9 SCLC samples, and 5 normal lung tissues [9]. GSE26939 dataset comprises of 116 adenocarcinoma subtype of NSCLC samples performed on the Agilent-UNC-custom4X44K platform [10]. GSE30219 dataset, performed on the Affymetrix Human Genome U133 Plus 2.0 Array platform, comprises 272 NSCLC samples, 21 SCLC samples, and 14 non-tumoral lung samples [11].

Follow-up overall survival data and common clinicopathological characteristics such as tumor type, stage, were available in all the three cohorts. Three major genetic changes, p53, EGFR and K-ras, were investigated in GSE11969 and GSE26939. GSE30219 dataset also has disease-free survival follow-up data of patients.

Identification of kinase genes significantly associated with poor outcome. Processed microarray data from all datasets were obtained from the GEO database, and analyzed using the BRB-ArrayTools v.4.4.0 developed by Dr. Richard Simon and BRB-ArrayTools Development Team [12]. After reducing multiple probes sets to one per gene symbol using the most variable probe measured by IQR across arrays, all the three datasets were filtered by a list of previously reported 522 kinase genes [6]. We computed each of these 522 kinase genes based on univariate Cox proportional hazards models and identified genes whose expression is correlated with survival time at a statistical significance level $(\mathrm{P}<0.05)$. We also perform univariate permutation tests during the survival analysis to control false discoveries.

Construction of a kinase gene signature model to evaluate the risk score of NSCLC patients. Two kinase genes cyclin-dependent kinase 2 (CDK2) and p21 protein (Cdc42/ Rac)-activated kinase 4 (PAK4) were identified to be survival predictors in both GSE11969 and GSE26939 cohorts. Then we performed a survival risk prediction using these two genes by BRB-ArrayTools. This tool calculated a risk-score for each sample based on a linear combination of the expression levels of CDK2 and PAK4 weighted by the regression coefficient derived from the univariate Cox regression analysis. The patients were classified into high- and low- risk subgroups based on their risk scores above or below the median values across all samples in each cohort.

In silico validation of the two kinase gene signature in an independent expression microarray dataset. The 2-gene predictor model was further directly applied to another independent NSCLC gene expression dataset GSE30219. The coefficients and the thresholds of GSE30219 dataset were determined by the same methods used in the training datasets GSE11969 and GSE26939. After grouping by the risk scores, the prognostic values of 2 kianse gene signature and single kinases in all these three cohorts were compared.

Patients and specimens for experimental validation of the 2 kinase gene signature. A series of clinical NSCLC samples were used to further confirm the predictive power of this 2-gene signature experimentally. A total of 96 cases of archival formalin-fixed, paraffin-embedded NSCLC tissues were used for immunohistochemical staining to evaluate CDK2 and PAK4 expression at protein levels. All the patients received treatment according to the National Comprehensive Cancer Network (NCCN) Clinical Practice Guidelines. The follow-up, defined as the time between surgical resection and cancer-related death, ranged from 6 months to 5 years. The specimens were used with informed consents, and the procedures of this study were approved by the research ethics committee of our institutes.

Immunohisitochemisstry (IHC) analyses of CDK2 and PAK4 expression in lung cancer specimens. Immunostaining was performed using a streptavidin-biotin-peroxidase complex kit (Dako, Copenhagen, Denmark). $4 \mu \mathrm{m}$ of sections were deparaffinized, and endogenous peroxidases were quenched in $3 \% \mathrm{H} 2 \mathrm{O} 2$. After microwave-citrate antigen retrieval in $10 \mathrm{mM}$ citrate buffer ( $\mathrm{pH}$ 6.0) for 60 minutes, sections were stained with rabbit antibodies anti-human CDK2 (Santa Cruz, 1: 100) and PAK4 (Santa Cruz, 1: 100) overnight at $4^{\circ} \mathrm{C}$. Detection was performed using diaminobenzidine as the chromogen, and was then counterstained with hematoxylin. Nonspecific rabbit immunoglobulin $\mathrm{G}$ was used as the negative control. Immunohistochemical scoring for each sample was done by the $\mathrm{H}$ score system by summing the intensity of staining weighted by the percentage of tumor staining at each intensity. The median $\mathrm{H}$ scores for immunohistostaining were used as a cutoff to divide patients into high ( $\geq$ median $\mathrm{H}$-score) and low subgroups (<median H-score) as described previously [13].

Statistics. Difference in survival between high- and lowrisk subgroups defined by single markers or combined gene signature was compared using the Kaplan-Meier curve method and log-rank test. The association between risk class and clincopathological characteristics was evaluated by chi-square test. Multivariate models were computed using Cox proportionalhazards regression. All the analyses were performed using GraphPad Prism 5.0 software.

\section{Results}

CDK2 and PAK4 reproducibly predict survival of NSCLC in two microarray cohorts. The prognostic powers of a complete of 528 kinase genes as published by Manning et al. [6] were compared in two independent microarray cohorts of NSCLC. We found that 50 genes in GSE11929 and 41 genes 
in GSE26939 were associated with survival at 0.05 level of the permutation univariate test. Among them, we found that two overlapping kinases CDK2 and PAK4 were significantly associated with poor survival in both datasets (Table 1), demonstrating a more robust and reproducible predictive value for the survival of NSCLC patients than the other kinase genes. However, these two kinases showed significant prognostic independence only in one cohort GSE11929, failed to retain independent of other clinical characteristics in GSE26939. Based on the weighted expression levels of each kinase, patients were classified into high- and low- subgroups. As seen in Table 2 , in both datasets, high CDK2 expression was significantly associated with poor differentiation status, but not with age, gender, stage. There is no significant association between PAK4 and clinical characteristics observed (Table 2).

Construction of 2 kinase gene signature for the survival prediction of NSCLC. Furthermore, we found that CDK2 and PAK4 are not significantly associated with each other ( $\mathrm{P}=0.0598$ in GSE11969; $\mathrm{P}=0.1370$ in GSE26939) in both datasets, suggesting that combination of these two markers would increase their prognostic effect on clinical outcome in patients with NSCLC. Therefore, we developed the 2-gene model by combining the weighted CDK2 and PAK4 expression to score the risk of tumors, which can divide patients into high- and low- risk subgroups. As seen in Figure 1A and $1 \mathrm{~B}$, the high-risk subgroups defined by the 2 -gene model had a significantly shorter survival in both GSE11929 and GSE26939 datasets. Moreover, the this CDK2-PAK4 signature had a superior prognostic significance in comparison with each single gene (Table 2). As seen in Table 2. we have not found clinicopathological relevance of the 2-gene signature in these two training CRC cohorts.

Validation of the prognostic potential of CDK2-PAK4 gene signature in GSE30219 dataset. To evaluate its prognostic potential, we further applied the 2-gene signature in another NSCLC microarray dataset GSE30219. Similar with the training datasets, 2-gene signature showed a higher hazard ratio (HR) for survival than the individual genes, and retained as independent prognostic factor in multivariate Cox regression model analysis. (Table 1, Figure 1C). Meanwhile, we also found that this 2-gene signature also provided independent and significant information on disease-free survival for the patients in the validation dataset. Good-risk and poor-risk subgroups defined by this 2 -gene classifier are associated with significantly different diseases-free survival (adjusted HR $=1.7544,95 \% \mathrm{CI}=$ 1.1224 to $2.7421, \mathrm{P}=0.0141$ ) (Figure 1D). We also validated that CDK2 and PAK4 expression was not correlated with

Table 1. Univariate and multivariate analysis for overall survival in three microarray datasets of non-small cell lung cancer and our immunohistochemistry experiment

\begin{tabular}{|c|c|c|c|c|}
\hline \multirow[t]{2}{*}{ Prognostic factor } & \multicolumn{2}{|c|}{ Univariate analysis } & \multicolumn{2}{|c|}{ Multivariate analysis } \\
\hline & HR $(95 \%$ CI $)$ & $P$ value & HR (95\% CI) & $P$ value \\
\hline \multicolumn{5}{|l|}{ Training 1 (GSE11929) } \\
\hline Grade & $1.4893(0.9956-2.2277)$ & 0.0538 & $1.3746(0.9181-2.0580)$ & 0.1242 \\
\hline Stage & $1.9902(1.5384-2.5747)$ & $<0.0001$ & $1.8870(1.3427-2.6519)$ & 0.0003 \\
\hline $\mathrm{CDK} 2$ & $1.6430(1.0370-2.6030)$ & 0.0316 & $1.6277(1.0039-2.6392)$ & 0.0493 \\
\hline PAK4 & $1.5842(1.0024-2.5037)$ & 0.0476 & $2.6360(1.3315-5.2185)$ & 0.0056 \\
\hline 2-gene signature & $1.8173(1.1506-2.8705)$ & 0.0107 & $2.9025(1.4871-5.6649)$ & 0.0019 \\
\hline \multicolumn{5}{|l|}{ Training 2 (GSE26939) } \\
\hline Grade & $1.3206(0.8731-1.9974)$ & 0.1900 & $1.2670(0.7734-2.0756)$ & 0.3498 \\
\hline Stage & $1.2811(0.9619-1.7062)$ & 0.0918 & $1.1885(0.8806-1.6041)$ & 0.2614 \\
\hline $\mathrm{CDK}$ & $1.8168(1.1147-2.9610)$ & 0.0165 & $1.6517(0.8535-3.1965)$ & 0.1383 \\
\hline PAK4 & $1.8462(1.1302-3.0157)$ & 0.0130 & $1.6847(0.9095-3.1205)$ & 0.0989 \\
\hline 2-gene signature & $2.0496(1.2457-3.3723)$ & 0.0033 & $2.0531(1.0508-4.0112)$ & 0.0362 \\
\hline \multicolumn{5}{|l|}{ Validation (GSE30219) } \\
\hline Stage & $1.6464(1.4112-1.9208)$ & $<0.0001$ & $1.4942(1.2594-1.7729)$ & $<0.0001$ \\
\hline CDK2 & $1.9826(1.4744-2.6658)$ & $<0.0001$ & $1.5814(1.1328-2.2078)$ & 0.0074 \\
\hline PAK4 & $1.1995(0.8938-1.6096)$ & 0.2216 & $0.9172(0.6692-1.2572)$ & 0.5931 \\
\hline 2-gene signature & $2.1019(1.5621-2.8282)$ & $<0.0001$ & $1.7025(1.2193-2.3772)$ & 0.0019 \\
\hline \multicolumn{5}{|c|}{ Immunohistochemistry experiment } \\
\hline Grade & $1.1995(0.7340-1.9602)$ & 0.4703 & $1.0584(0.6322-1.7720)$ & 0.8300 \\
\hline Stage & $3.1592(1.6411-6.0818)$ & 0.0006 & $2.5403(1.2927-4.9920)$ & 0.0071 \\
\hline $\mathrm{CDK} 2$ & $2.0224(1.0529-3.8848)$ & 0.0293 & $1.9747(1.0078-3.8694)$ & 0.0486 \\
\hline PAK4 & $1.9112(1.0032-3.6411)$ & 0.0532 & $1.6241(0.8202-3.2157)$ & 0.1663 \\
\hline CDK2/PAK4 & $1.4554(1.0870-1.9487)$ & 0.0122 & $1.3441(1.0034-1.8005)$ & 0.0485 \\
\hline 2-gene signature (weighted) & $3.2914(1.7126-6.3256)$ & 0.0003 & $2.8230(1.3725-5.8065)$ & 0.0050 \\
\hline
\end{tabular}



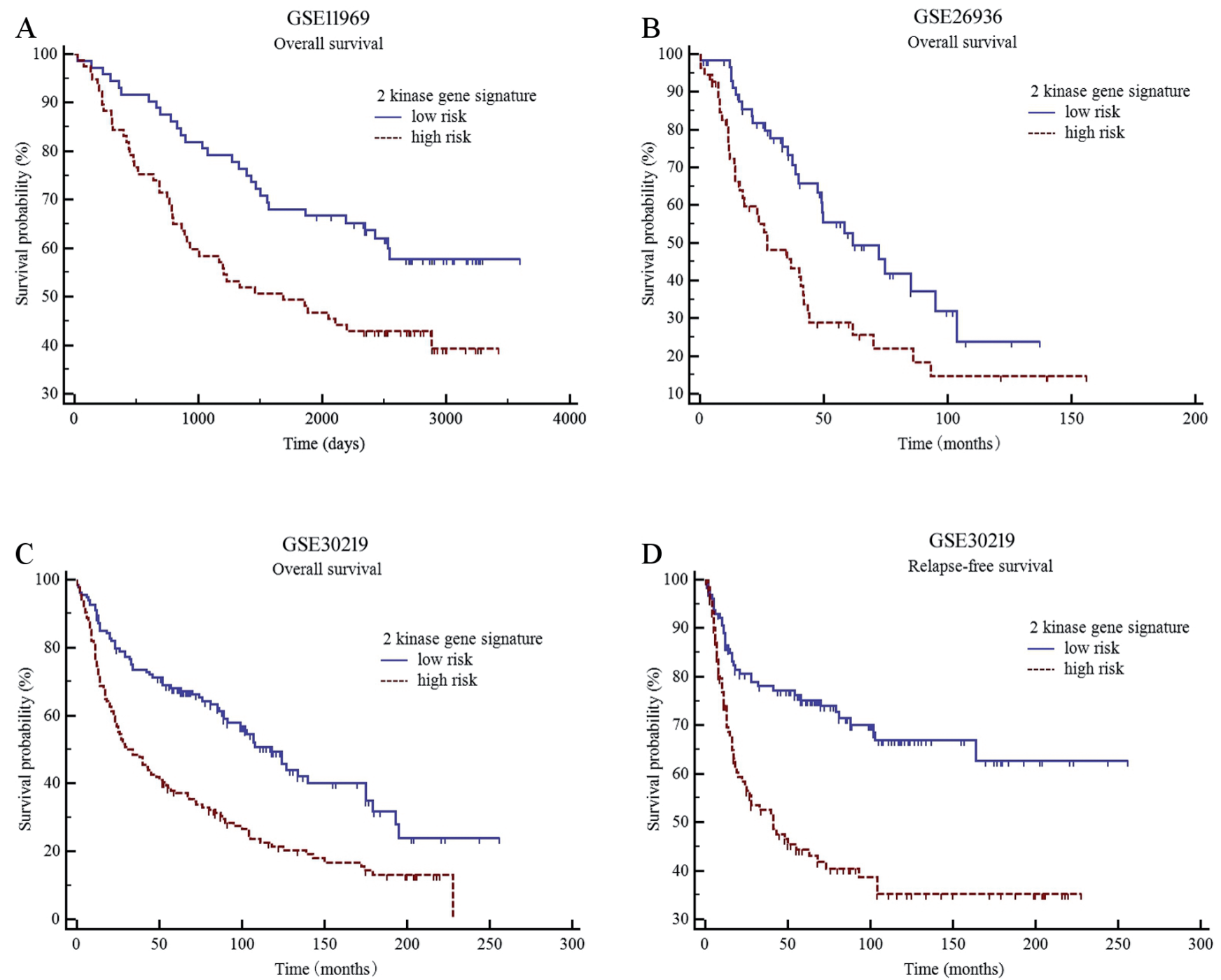

Figure 1. Kaplan-Meier curves of overall survival and disease-free survival in terms of 2-gene signature. This 2-gene signature can stratify patients into two subgroups with significant differences in overall survival in GSE11929 (A), GSE26936 (B), and GSE30219 (C), disease-free survival in GSE30219 (D).

each other. Different from the training cohorts, 2-gene signature had a significant clinicopathological relevance with stage and gender (Table 2).

Experimental validation of the prognostic potential of CDK2-PAK4 gene signature in a NSCLC cohort. We next experimentally detected the CDK2-PAK4 signature expression by IHC separately in a cohort of NSCLC samples. Representative images of IHC staining for CDK2 and PAK4 were shown in Figure 2. Similar with the microarray datasets, there was no significant association observed between CDK2 and PAK4 expression. We adopted two stratification approaches to evaluate the prognostic potential of CDK2-PAK4 signature. The first stratification strategy is performed using the median $\mathrm{H}$ score as a cutoff. Samples were divided into high- and low-expression subgroups. Our immunohistochemical results showed a strong correlation between high CDK2 expression with survival independent of other clinically employed predictors, but PAK4 only had a marginal significance level (Table 1). When two protein markers are combined, patients were classified into 4 subgroups: high-CDK2/high-PAK4, high-CDK2/low-PAK4, low-CDK2/high-PAK4, and low-CDK2/low-PAK4. As seen in Figure $3 \mathrm{~A}$ and Table 1, high-CDK2/high-PAK4 subgroups had the worst prognosis, low-CDK2/low-PAK4 groups had the best clinical outcome. The second stratification strategy is performed using the 2 gene signature model based on a summation of weighted CDK2 and PAK4 expression as used in the microarray dataset analysis. As seen in Figure 3B, IHC assay of this model can recapitulate the risk classifications effect of this 2 gene signature model in micrarray datasets. 

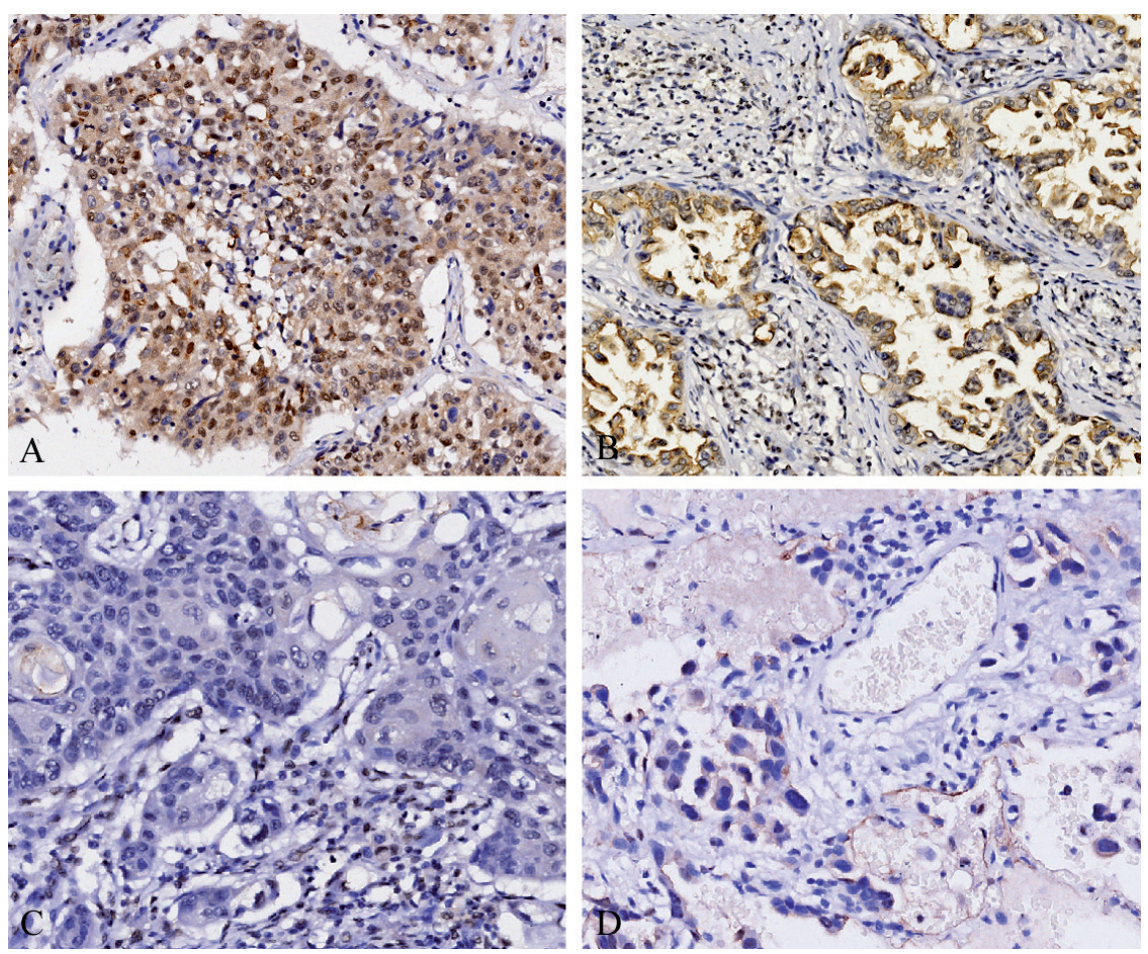

Figure 2. Representative images of CDK2 and PAK4 expression in non-small lung cancers. (A) high expression of CDK2 with nuclear staining; (B) high expression of PAK4 with cytocytoplasmic staining; (C) low expression of CDK2; (D) low expression of PAK4. High and low expression of immunostaining was subgrouped using the median $\mathrm{H}$-score as a cutoff.

When included in multivariate survival analyses with standard clinical pathological information including grade and stage, this 2-gene classifier retained to be a significant and independent predictor of survival in our experimental cohorts (Table 1). Furthermore, our immunohistochemistry experiment had not found significant association between single or combined CDK2 and PAK4 expression with clinicopathological characteristics in CRC patients (Table 2).
A

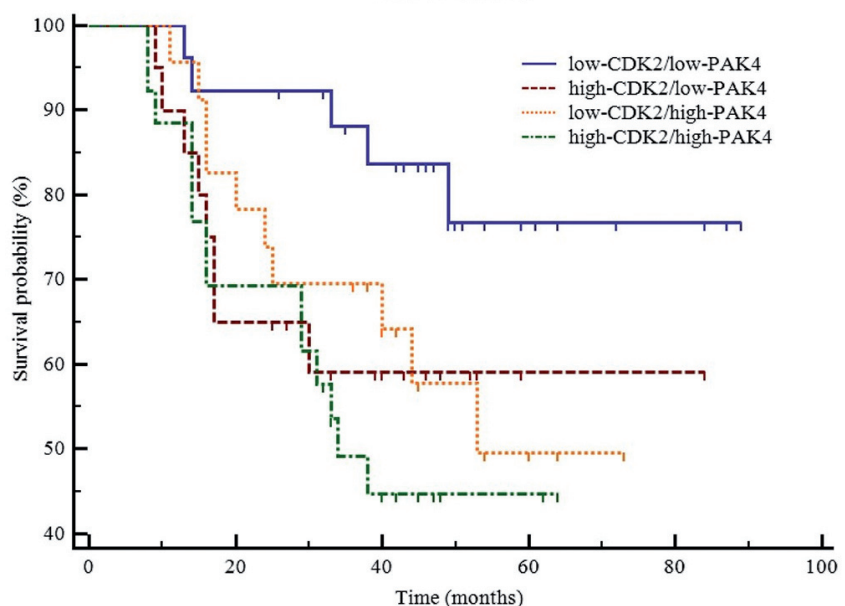

B

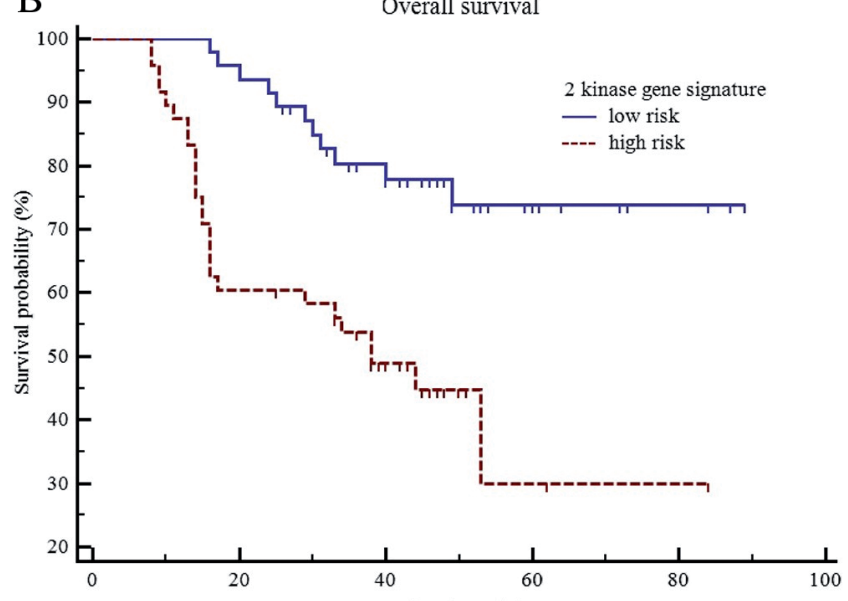

Figure 3. Immunohistochemistry (IHC) assay of protein pattern of CDK2-PAK4 gene signature in non-small lung cancer samples. (A) Combined CDK2 and PAK4 classified tumors into four subgroups with difference in survival. (B) 2-gene model using weighted CDK2-PAK4 expression recapitulate the risk classifications effect in micrarray datasets. 
Table 2. Relationship between CDK2 and PAK4 expression with clinical characteristics in three microarray datasets of non-small cell lung cancer and our immunohistochemistry experiment

\begin{tabular}{|c|c|c|c|c|c|c|c|c|c|}
\hline \multirow[b]{2}{*}{ Characteristics } & \multicolumn{3}{|c|}{ CDK2 expression } & \multicolumn{3}{|c|}{ PAK4 expression } & \multicolumn{3}{|c|}{ 2-gene signature } \\
\hline & low & high & $\mathbf{P}$ & low & high & $\mathbf{P}$ & low & high & $\mathbf{P}$ \\
\hline \multicolumn{10}{|l|}{ GSE11969 } \\
\hline \multicolumn{10}{|l|}{ Age } \\
\hline$<65$ & 46 & 41 & 0.8574 & 42 & 45 & 0.6676 & 41 & 46 & 0.8574 \\
\hline$\geq 65$ & 31 & 31 & & 33 & 29 & & 31 & 31 & \\
\hline \multicolumn{10}{|l|}{ Gender } \\
\hline Male & 47 & 54 & 0.0996 & 53 & 48 & 0.5603 & 48 & 53 & 0.9147 \\
\hline Female & 30 & 18 & & 22 & 26 & & 24 & 24 & \\
\hline \multicolumn{10}{|l|}{ Grade } \\
\hline 1 & 20 & 5 & & 9 & 16 & & 16 & 9 & \\
\hline 2 & 23 & 8 & 0.0028 & 17 & 14 & 0.3540 & 17 & 14 & 0.3117 \\
\hline 3 & 14 & 20 & & 17 & 17 & & 15 & 19 & \\
\hline N.A. & 20 & 39 & & 32 & 27 & & 24 & 35 & \\
\hline \multicolumn{10}{|l|}{ Stage } \\
\hline $1-2$ & 54 & 50 & 0.9303 & 54 & 50 & 0.6812 & 51 & 53 & 0.9303 \\
\hline $3-4$ & 23 & 22 & & 21 & 24 & & 21 & 24 & \\
\hline \multicolumn{10}{|l|}{ GSE26939 } \\
\hline \multicolumn{10}{|l|}{ Age } \\
\hline$<65$ & 31 & 21 & 0.0417 & 26 & 26 & 0.8767 & 33 & 19 & 0.0533 \\
\hline$\geq 65$ & 24 & 38 & & 29 & 33 & & 27 & 35 & \\
\hline \multicolumn{10}{|l|}{ Gender } \\
\hline Male & 23 & 29 & 0.5502 & 23 & 29 & 0.5502 & 22 & 30 & 0.0667 \\
\hline Female & 32 & 30 & & 32 & 30 & & 38 & 24 & \\
\hline \multicolumn{10}{|l|}{ Grade } \\
\hline 1 & 5 & 3 & & 4 & 4 & & 5 & 3 & \\
\hline 2 & 34 & 24 & 0.0250 & 29 & 29 & 0.9386 & 35 & 23 & 0.0979 \\
\hline 3 & 14 & 29 & & 20 & 23 & & 17 & 26 & \\
\hline N.A. & 2 & 3 & & 2 & 3 & & 3 & 2 & \\
\hline \multicolumn{10}{|l|}{ Stage } \\
\hline $1-2$ & 39 & 41 & & 36 & 44 & & 44 & 36 & \\
\hline $3-4$ & 7 & 14 & 0.3095 & 11 & 10 & 0.7206 & 8 & 13 & 0.2567 \\
\hline N.A. & 12 & 6 & & 7 & 5 & & 8 & 5 & \\
\hline \multicolumn{10}{|l|}{ GSE30219 } \\
\hline Age & & & & & & & & & \\
\hline$<65$ & 86 & 72 & 0.0906 & 75 & 83 & 0.4249 & 83 & 75 & 0.3445 \\
\hline$\geq 65$ & 47 & 62 & & 58 & 51 & & 50 & 59 & \\
\hline N.A. & 1 & 0 & & 0 & 1 & & 1 & 0 & \\
\hline Gender & & & & & & & & & \\
\hline Male & 105 & 121 & 0.0117 & 114 & 112 & 0.6517 & 106 & 120 & 0.0289 \\
\hline Female & 29 & 13 & & 19 & 23 & & 28 & 14 & \\
\hline Stage & & & & & & & & & \\
\hline $1-2$ & 116 & 92 & 0.0005 & 110 & 98 & 0.0823 & 115 & 93 & 0.0013 \\
\hline $3-4$ & 17 & 42 & & 23 & 36 & & 18 & 41 & \\
\hline N.A. & 1 & 0 & & 0 & 1 & & 1 & 0 & \\
\hline Immunohistoch & rimen & & & & & & & & \\
\hline Age & & & & & & & & & \\
\hline$<65$ & 26 & 23 & 0.8425 & 23 & 26 & 0.8415 & 35 & 28 & 0.1160 \\
\hline$\geq 65$ & 23 & 24 & & 24 & 23 & & 12 & 21 & \\
\hline Gender & & & & & & & & & \\
\hline Male & 33 & 38 & 0.2025 & 31 & 40 & 0.1293 & 32 & 39 & 0.2930 \\
\hline Female & 16 & 9 & & 16 & 9 & & 15 & 10 & \\
\hline Grade & & & & & & & & & \\
\hline 1 & 7 & 5 & & 7 & 5 & & 7 & 5 & \\
\hline 2 & 22 & 27 & 0.4684 & 23 & 26 & 0.7772 & 21 & 28 & 0.4608 \\
\hline 3 & 20 & 15 & & 17 & 18 & & 19 & 16 & \\
\hline Stage & & & & & & & & & \\
\hline $1-2$ & 33 & 30 & 0.8825 & 33 & 30 & 0.4765 & 35 & 28 & 0.1160 \\
\hline $3-4$ & 16 & 17 & & 14 & 19 & & 12 & 21 & \\
\hline
\end{tabular}




\section{Discussion}

Although there is an increasing awareness that the dysregulation of kinase genes is associated with poor clinical outcome in NSCLC, previous studies have not address which gene or gene signature have a strong and reproducible prognostic capacity. In this study, from the kinase list proposed, 2 genes, CDK2 and PAK4 were identified as lung cancer survival factors across independent datasets, which is consistent with the previous findings. Moreover, our in silico and experimental evidence support that a 2-gene signature combining CDK2 and PAK4 has a higher predictive prognostic performance than the single kinases, that have not been previously recognized. Our results further indicates that the prognostic power would increase substantially when the 2 -gene predictor is combined with existing outcome predictors. Therefore, we suggest this 2 kinase gene signature could be used to identify patients at high risk for poor outcome after standard therapy, which might benefit from more intensive or innovative treatment.

The prognostic significance of individual CDK2 and PAK4 gene has been well established. Previous studies indicated a robust relationship between immunostaining results and qRT-PCR expression values for CDK2 and PAK4 [14-16]. In this study, we used IHC to evaluate the protein pattern of the 2-gene signature for lung cancer, and reproduced the risk classification effect at mRNA level. Therefore, another benefit of this 2-gene signature is that its clinical use could be fast, reproducible, automatable, and particularly, can be performed on archived formalin-fixed, paraffin-embedded tissue (FFPE) samples. The 2-gene signature demonstrated unconsistent clinical relevance in microarray datasets, but not in our experiment data. These difference may originate from the discrepancies in experimental platforms and sample sizes in different studies.

Functionally, CDK2 and PAK4 have been identified to important regulators in lung cancer. Recently, Hu et al. [17] found that CDK2 can mediate anaphase catastrophe in lung cancer cells. Cai et al. [16] indicated that PAK4 mediated LIMK1 phosphorylation regulates the migration and invasion in NSCLC. CDK2 is regarded as potential targets for cancers; several CDK2 inhibitors have entered clinical trials [18]. Currently, no study investigate the direct interaction of CDK2 and PAK4. However, interestingly, the previous studies indicated that all these two kinases can phosphorylate a transforming growth factor-beta (TGF- $\beta$ ) signaling effector Smad2 and attenuate $\mathrm{Smad} 2$ transactivation, thus transcriptional regulation $[19,20]$. Therefore, we speculate that CDK2 and PAK4 may co-target the tumor suppressor Smad2, and thus exert a disruption effect of TGF- $\beta$ signaling and contribute to the development and progression of CRC.

Most recently, a PAK4 inhibitor, PF-3758309, has been developed, showed a significant anti-metastatic effect in human lung cancer cell via regulation of CREB, NF- $\mathrm{KB}$, and $\beta$-catenin signalings [21]. Therefore, we propose that this 2-gene kinase gene signature is of important therapeutic value for NSCLC, the high-risk patients stratified by this classifier may benefit from inhibitors against CDK2 and PAK4.

In conclusion, in this study, we established a two kinase gene signature for outcome risk stratification in lung cancer. This 2-gene classifier reproducibly yields a better prognostic performance than the individual kianses, and can add significant prognostic information to the existing outcome risk factors. We also proposed that poor outcome patients with NSCLC defined by this kinase pair might be the potential candidates for the CDK2 and PAK4 inhibitors.

Acknowledgments: This study was supported by the Zhejiang Province Welfare Technology Applied Research Program (NO. 2014C33245), Medicine and Health Project of Zhejiang Province (No. 2015RCB028), and Huzhou Science and Technology Fund (NO. 2014GZ11).

\section{References}

[1] CHEN HY, YU SL, CHEN CH, CHANG GC, CHEN CY, et al. A five-gene signature and clinical outcome in non-small-cell lung cancer. N Engl J Med. 2007; 356: 11-20. http://dx.doi. org/10.1056/NEJMoa060096

[2] BOUTROS PC, LAU SK, PINTILIE M, LIU N, SHEPHERD FA, et al. Prognostic gene signatures for non-small-cell lung cancer. Proc Natl Acad Sci U S A. 2009; 106: 2824-8. http:// dx.doi.org/10.1073/pnas.0809444106

[3] PLANCK M, ISAKSSON S, VEERLA S, STAAF J. Identification of transcriptional subgroups in EGFR-mutated and EGFR/KRAS wild-type lung adenocarcinoma reveals gene signatures associated with patient outcome. Clin Cancer Res. 2013; 19: 5116-26. http://dx.doi.org/10.1158/1078-0432.CCR13-0928

[4] SUBRAMANIAN J, SIMON R. Gene expression-based prognostic signatures in lung cancer: ready for clinical use? J Natl Cancer Inst. 2010; 102: 464-74. http://dx.doi.org/10.1093/ jnci/djq025

[5] ZHANG J, YANG PL, GRAY NS. Targeting cancer with small molecule kinase inhibitors. Nat Rev Cancer. 2009; 9: 28-39. http://dx.doi.org/10.1038/nrc2559

[6] MANNING G, WHYTE DB, MARTINEZ R, HUNTER T, SUDARSANAM S. The protein kinase complement of the human genome. Science. 2002; 298: 1912-34. http://dx.doi. org/10.1126/science.1075762

[7] YANG Y, LUO J, ZHAI X, FU Z, TANG Z, et al. Prognostic value of phospho-Akt in patients with non-small cell lung carcinoma: a meta-analysis. Int J Cancer. 2014; 135: 1417-24. http://dx.doi.org/10.1002/ijc.28788

[8] FINOCCHIARO G, TOSCHI L, GIANONCELLI L, BARETTI M, SANTORO A. Prognostic and predictive value of MET deregulation in non-small cell lung cancer. Ann Transl Med. 2015; 3: 83.

[9] TAKEUCHI T, TOMIDA S, YATABE Y, KOSAKA T, OSADA $\mathrm{H}$, et al. Expression profile-defined classification of lung adenocarcinoma shows close relationship with underlying 
major genetic changes and clinicopathologic behaviors. J Clin Oncol. 2006; 24: 1679-88. http://dx.doi.org/10.1200/ LCO.2005.03.8224

[10] WILKERSON MD, YIN X, WALTER V, ZHAO N, CABANSKI CR, et al. Differential pathogenesis of lung adenocarcinoma subtypes involving sequence mutations, copy number, chromosomal instability, and methylation. PLoS One. 2012; 7: e36530. http://dx.doi.org/10.1371/ journal.pone.0036530

[11] ROUSSEAUX S, DEBERNARDI A, JACQUIAU B, VITTE AL, VESIN A, et al. Ectopic activation of germline and placental genes identifies aggressive metastasis-prone lung cancers. Sci Transl Med. 2013; 5: 186ra66. http://dx.doi.org/10.1126/ scitranslmed.3005723

[12] SIMON R, LAM A, LI MC, NGAN M, MENENZES S, et al. Analysis of gene expression data using BRB-ArrayTools. Cancer Inform. 2007; 3: 11-7.

[13] HUANG HJ, NEVEN P, DRIJKONINGEN M, PARIDAENS $\mathrm{R}$, WILDIERS $\mathrm{H}$, et al. Association between tumour characteristics and HER-2/neu by immunohistochemistry in 1362 women with primary operable breast cancer. J Clin Pathol. 2005; 58: 611-6. http://dx.doi.org/10.1136/ jcp.2004.022772

[14] VOLM M, KOOMAGI R. Relevance of proliferative and proapoptotic factors in non-small-cell lung cancer for patient survival. Br J Cancer. 2000; 82: 1747-54.

[15] DOBASHI Y, SHOJI M, JIANG SX, KOBAYASHI M, KAWAKUBO Y, et al. Active cyclin A-CDK2 complex, a possible critical factor for cell proliferation in human primary lung carcinomas. Am J Pathol. 1998; 153: 963-72. http://dx.doi. org/10.1016/S0002-9440(10)65638-6

[16] CAI S, YE Z, WANG X, PAN Y, WENG Y, et al. Overexpression of $\mathrm{P} 21$-activated kinase 4 is associated with poor prognosis in non-small cell lung cancer and promotes migration and invasion. J Exp Clin Cancer Res. 2015; 34: 48. http:// dx.doi.org/10.1186/s13046-015-0165-2

[17] HU S, DANILOV AV, GODEK K, ORR B, TAFE LJ, et al. CDK2 Inhibition Causes Anaphase Catastrophe in Lung Cancer through the Centrosomal Protein CP110. Cancer Res. 2015; 75: 2029-38. http://dx.doi.org/10.1158/0008-5472. CAN-14-1494

[18] WADLER S. Perspectives for cancer therapies with cdk2 inhibitors. Drug Resist Updat. 2001; 4: 347-67. http://dx.doi. org/10.1054/drup.2001.0224

[19] BAUGHN LB1, DI LIBERTO M, NIESVIZKY R, CHO HJ, JAYABALAN D, et al. CDK2 phosphorylation of Smad2 disrupts TGF-beta transcriptional regulation in resistant primary bone marrow myeloma cells. J Immunol. 2009; 182: 1810-7. http://dx.doi.org/10.4049/jimmunol.0713726

[20] WANG C, LI Y, ZHANG H, LIU F, CHENG Z, et al. Oncogenic PAK4 regulates Smad2/3 axis involving gastric tumorigenesis. Oncogene. 2014; 33: 3473-84. http://dx.doi. org/10.1038/onc. 2013.300

[21] MURRAY BW, GUO C, PIRAINO J, WESTWICK JK, ZHANG C, et al. Small-molecule p21-activated kinase inhibitor PF-3758309 is a potent inhibitor of oncogenic signaling and tumor growth. Proc Natl Acad Sci U S A. 2010; 107: 9446-51. http://dx.doi.org/10.1073/pnas.0911863107 